\title{
Effects of Crystal Pixel Size and Collimator Geometry on the Performance of a Pixelated Crystal $\gamma$-Camera Using Monte Carlo Simulation
}

\author{
Mahmoud Rasouli ${ }^{1}$, Abbas Takavar ${ }^{1}$, Mohammad Reza Ay ${ }^{1,2}$, Sasan Saber $^{3}$, and George Loudos ${ }^{4}$ \\ ${ }^{I}$ Medical Physics and Biomedical Engineering Department, School of Medicine, Tehran University of Medical Sciences, Tehran, Iran; \\ ${ }^{2}$ Research Centre for Science and Technology in Medicine, Tehran University of Medical Sciences, Tehran, Iran; ${ }^{3}$ Pulmonary Unit of \\ Shariati Hospital, Tehran University of Medical Sciences, Tehran, Iran; and ${ }^{4}$ Department of Medical Instruments Technology, \\ Technological Educational Institutes of Athens, Athens, Greece
}

Dedicated $\gamma$-cameras based on pixelated scintillators have long been used for breast tumor imaging. Intercrystal scattering (ICS) increases the background counting rate and degrades the image quality when small crystal pixels are used. Because of the small size of applied collimators, scattered radiation and septal penetration are high, and therefore collimator characteristics must be carefully considered. In our study, we investigated the influence of ICS events on position-detection accuracy (PDA) for pixelated crystals and the effects of different geometries of hexagonal-hole collimators on the performance of these cameras, using Monte Carlo simulation to optimize camera design. The arrays of thallium-doped cesium iodide detectors with different pixel dimensions that had been exposed to $140-\mathrm{keV}$ photons of isotropic point source, $50 \mathrm{~mm}$ from the collimator surface, were simulated. Hexagonal-hole collimators were $10.5,15$, and $21 \mathrm{~mm}$ long. The septal thickness varied from 0.1 to $0.5 \mathrm{~mm}$, with 3 different hole diameters. The results confirmed that by increasing the crystal pixel size, ICS was decreased and change of detection efficiency was negligible, but PDA, contrast-to-noise ratio, and spatial resolution (full width at half maximum) were increased. Our experiences confirmed that $2 \times 2 \mathrm{~mm}$ was an optimum crystal pixel size, especially for a lower ICS fraction and an appropriate full width at half maximum. Because collimators are the limiting factor for spatial resolution and sensitivity, careful collimator design is of great importance.

Key Words: pixelated $\gamma$-camera; PDA; DE; ICS; CNR

J Nucl Med Technol 2010; 38:199-204

DOI: 10.2967/jnmt.109.073072

\section{$\mathbf{S}$} andard $\gamma$-cameras traditionally have been used to diagnose a major health problem for women - that is, breast carcinoma. System limits are essentially related to the spa-

\footnotetext{
Received Nov. 28, 2009; revision accepted Sep. 17, 2010.

For correspondence contact: Mahmoud Rasouli, Medical Physics and Biomedical Engineering Department, School of Medicine, Tehran University of Medical Sciences, N. Kargar St., Poursina Ave., Tehran, Iran 1419853851. E-mail: m_rasouli@razi.tums.ac.ir

COPYRIGHT @ 2010 by the Society of Nuclear Medicine, Inc.
}

tial resolution of these standard $\gamma$-cameras, prompting a need for researchers to build dedicated high-resolution $\gamma$-cameras. The pixelated crystal, in comparison to continuous crystals, limits the degree to which the scintillation light spreads laterally and thereby can improve spatial resolution. Of course, such a design improves spatial resolution at the expense of a deterioration of the energy resolution resulting from light losses in the pixelated crystal, compared with that of a single, continuous crystal. However, the arrays of solid-state detectors, such as cadmium-zinc-telluride, provide a promising alternative technology as a compact high-resolution $\gamma$-camera, with the advantage of improved energy resolution, compared with the resolution of pixilated scintillators. Many workers up to now have performed simulations and measurements to improve the performance of these cameras for detecting T1a tumors (size, $<5 \mathrm{~mm}$ ) (1-7). Monte Carlo (MC) simulation techniques are useful when experimental measurements are not practical or experiments are too expensive. In an ideal environment, the parameters affecting the performance of an imaging system, such as collimator configuration and detector pixel size, are first optimized by means of accurate simulation, and then the optimized setup is tested experimentally (8). MCNP MC code is a well-known and generalpurpose code that permits the transport of photons and electrons in various medium (9). It is well known that intercrystal scattering (ICS) events increase the background counting rate and degrade image quality. Although ICS events are more important in PET with higher photon energy ranges (10-12), it appears that the small crystal size in high-resolution breast imaging systems causes these systems to suffer from ICS events, even when low-energy $(140-\mathrm{keV})$ photons are used. On the other hand, because collimators are the limiting factor for spatial resolution and sensitivity in $\gamma$-cameras, careful collimator design is crucial (13). In our study, the influence of crystal size on ICS events has been quantitatively calculated. The geometry of the collimator and the classic trade-off between the spatial resolution and sensitivity of the collimator are studied. 
Besides detection efficiency (DE) and position-detection accuracy (PDA), the performance of 3 different types of collimators in terms of contrast-to-noise ratio (CNR) is compared using MC simulation. Our object was to calculate the contribution of ICS events in 3 sizes of crystals and find the best crystal pixel size and geometry of hexagonalhole collimators to achieve higher PDA, CNR, and DE and lower full width at half maximum (FWHM) and ICS events. We performed these simulations without a collimator in a previous study and obtained good results (14). In this study, we wanted to determine the effects of a collimator on our results and camera performance.

\section{MATERIALS AND METHODS \\ MCNP4C MC Code}

In this study, we used the MCNP4C general-purpose MC code for accurate modeling of ICS effects in different crystal pixel sizes and to find the best geometry of hexagonalhole collimators that can be used in pixelated $\gamma$-cameras. For photon transport, the code takes into account photoelectric absorption, with the possibility of K- and L-shell fluorescent emission or Auger electrons, coherent and incoherent scattering, and pair production. The photoelectric cross sections are based on the work of Storm and Israel (15), and the scattering cross sections are taken from tabulations of the effective number of degrees of freedom (16). The continuous-slowing-down-approximation energy-loss model is used for electron transport. To improve the efficiency of electron and photon transport, 2 cards (PHYS:P and PHYS:E) were implemented in MCNP for biasing some physical parameters such as upper energy limit for electrons and photons (EMAX and EMCPF), production of secondary electrons by photons (IDES), coherent scattering $(\mathrm{NOCOH})$, production of photons by electrons (IPHOT), and production of characteristic $\mathrm{x}$-rays (XNUM). The geometry of MCNP treats an arbitrary 3-dimensional configuration in the Cartesian coordinate system. The number of cells in this code should be less than $10^{5}$. If this limit was exceeded when using cell geometry, the lattice geometry was used (9).

\section{Simulation Set-up}

In this study, we used as a pixelated scintillation crystal the different arrays of a cesium iodide scintillator-with surface areas of $36 \mathrm{~cm}^{2}$ and pixel dimensions of $3 \times 3 \times 5$, $2 \times 2 \times 5$, and $1 \times 1 \times 5 \mathrm{~mm}^{3}$ - which were exposed to $140-\mathrm{keV}$ photons from a ${ }^{99 \mathrm{~m}} \mathrm{Tc}$ point source in air as an orthogonal pencil beam to the center of the crystal. Detector pixels have no spacing between them. Lead hexagonalhole collimators with 3 hole diameters $(0.5,1$, and $1.5 \mathrm{~mm})$, 3 septal lengths $(10.5,15$, and $21 \mathrm{~mm}$ [as high-sensitivity, general-purpose, and high-resolution collimators, respectively], and septal thicknesses $(0.1,0.2,0.3,0.4$, and 0.5 $\mathrm{mm}$ ) were used. Source-to-collimator surface distance was set to $50 \mathrm{~mm}$, and the energy window applied in simulations was $20 \%(126-154 \mathrm{keV})$. A total of 50,000 photons from a point source irradiated the central crystal in each array. Because our assessment goal was to determine the relationship between ICS and crystal pixel size and the influence of collimator geometry on camera performance, the attenuation properties of the crystal and collimator were considered in the code. We also included septal penetration, scattering, and other physical effects inside the collimators. The debug information card (DBCN) in the MCNP4C code was set to write the detail information (energy, point of interaction, and direction of photons) about the interaction of photons with crystal arrays. Before starting, the code was validated through comparison with some experimental measurements. Figure 1 shows the code validation by comparison of our results and the results of spatial resolution in the study by Loudos and et al. (17).

\section{Definitions}

We considered PDA as the percentage of events that were correctly positioned in the irradiated crystal - the number of detected events in irradiated pixel versus all detected events in crystal. The position of detected photons in this study was calculated on the basis of Anger position logic. The ICS fraction was defined as the ratio of number of scattered photons, which deposit some energy in more than one detector array pixel, to the total number of detected photons. DE is defined as the ratio of number of detected events in the crystal to the number of photons emitted. Although there are several definitions of contrast, the most commonly used is absolute [(maximum - minimum)/ (maximum + minimum)], where maximum and minimum are pixel value within and outside some object to be detected. CNR is essentially the contrast in a lesion, such as just defined, divided by the noise (here expressed in terms of the coefficient of variation that is the SD divided by the mean, which is often expressed as a percentage) (18). Spatial resolution is equal to the FWHM of the collimator-detector response to the point source (19).

\section{RESULTS}

Table 1 shows the percentage of ICS events for a collimator with a septal thickness of $0.2 \mathrm{~mm}$ and different hole diameters, septal lengths, and crystal pixel sizes. Figure 2 shows DE for different collimator geometries. Table 2 shows the sensitivity data for collimators (pixel size of $4 \mathrm{~mm}^{2}$ ) of different geometries (cpm/MBq). The results of PDA, CNR, and spatial resolution (FWHM) for different crystal pixel sizes and collimator geometries are summarized in Tables 3, 4, and 5, respectively.

\section{DISCUSSION}

This work investigated the ICS effect at $140 \mathrm{keV}$ with orthogonal irradiation and the effects of collimator characteristics in high-resolution breast tumor imaging with detectors of small crystal pixel size. Although the transport of $140-\mathrm{keV}$ photons, using the MC method, was timeconsuming, our data provided detailed information about 


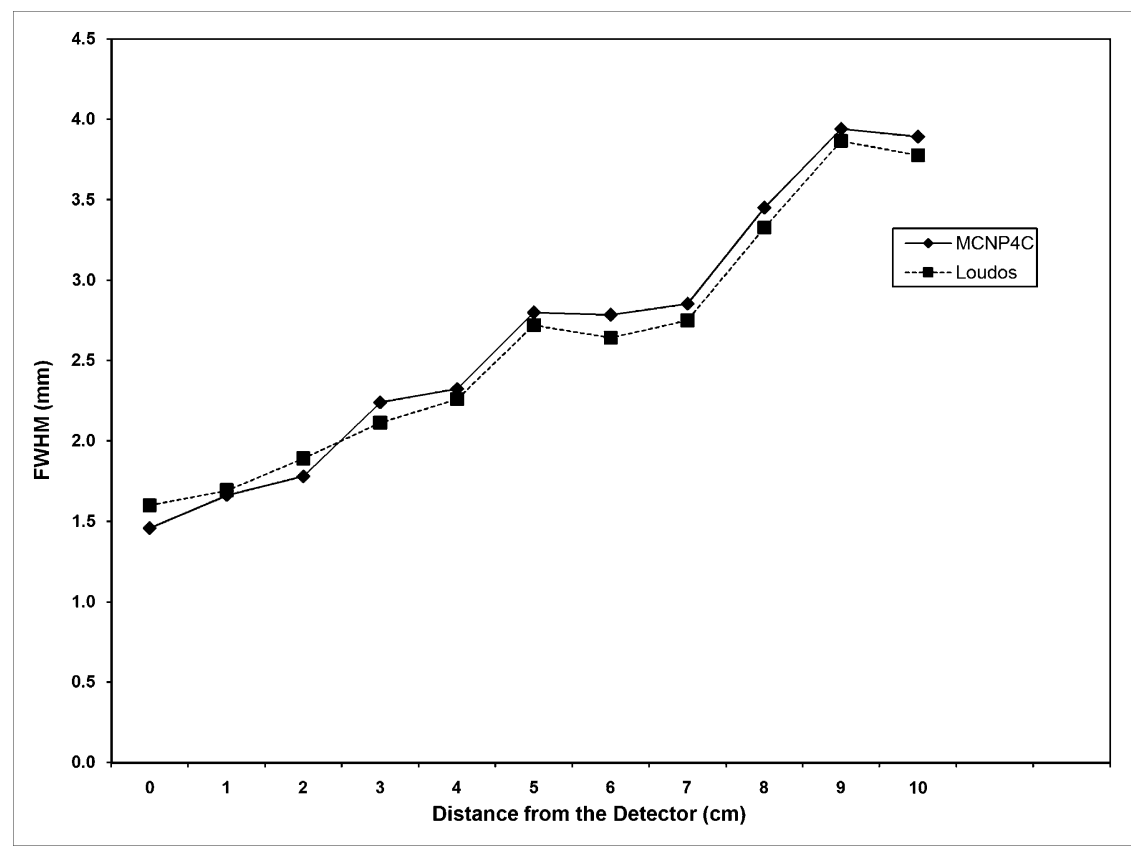

FIGURE 1. Comparison of results of spatial resolution (FWHM in $\mathrm{mm}$ ) between MCNP4C code simulation and experimental test of Loudos et al. (17). photon interaction within the collimator and crystal. This information can be useful for the accurate measurement and modeling of ICS effects (as a function of crystal dimensions) and designing of the best collimator geometry for higher-resolution imaging systems, which is being actively investigated by our group. Quantification of the contribution of ICS on detected events in our study showed that ICS events were increased when crystal pixel size was decreased. For smaller pixel sizes, Compton-scattered photons can exit from the irradiated pixel without any interaction with the adjacent pixel. As a result, ICS events were enhanced, and photon mispositioning was increased. Thus, an accurate optimization concerning pixel size and the contribution of ICS is necessary in high-resolution breast tumor imaging systems. The percentage of detected ICS events decreases when pixel size increases (Table 1). Collimator geometric configuration plays an important role. An increase in septal length (collimator thickness) caused a decrease in ICS events. A septal thickness of up to $0.2 \mathrm{~mm}$ drastically decreased ICS events. By increasing septal thickness, the corresponding decrease in ICS events was slower. A smaller hole diameter also caused fewer ICS events. ICS fraction in high-sensitivity collimators decreased from $0.34 \%$ to $0.16 \%$, from $2.33 \%$ to $1.26 \%$, and from $5.49 \%$ to $3.19 \%$ for hole diameters of $0.5,1$, and $1.5 \mathrm{~mm}$, respectively. DE was improved with an increase in hole diameter and a decrease in collimator septal length. Also for a septal thickness of up to $0.2 \mathrm{~mm}$, the rate of $\mathrm{DE}$ changes was severe and then decreased gradually. DE depends on crystal material (effective atomic number, stopping power, and photoelectric probability) and crystal thickness. According to these results, pixel size had a negligible effect on DE. In contrast, collimator geometry significantly affected this parameter.
By decreasing the hole diameter and increasing the septal length and septal thickness of the collimator, sensitivity is decreased (Table 2).

We used a weighted-energy method for photon interaction positioning in the scintillation crystal; thus, PDA was improved by the increase in pixel size (due to decreasing ICS events) and the consecutive reduction of mispositioned events. On the other hand, PDA was worsened by increasing collimator hole diameter and decreasing septal length. Also, PDA improved for a septal thickness of up to 0.2 $\mathrm{mm}$, and when septal thickness was increased further, PDA decreased. PDA is also affected by crystal thickness, but in this study the crystal thickness was constant. Another factor that affected PDA was radiation angle. In this work, we have used orthogonal radiation.

TABLE 1

Percentage of ICS Events in Collimators with Different Crystal Pixel Sizes, Hole Diameters, and Septal Lengths

\begin{tabular}{cclll}
\hline & & \multicolumn{3}{c}{ Pixel size $\left(\mathrm{mm}^{2}\right)$} \\
\cline { 4 - 5 } $\begin{array}{c}\text { Type of } \\
\text { collimator }\end{array}$ & $\begin{array}{c}\text { Hole diameter } \\
(\mathrm{mm})\end{array}$ & \multicolumn{1}{c}{1} & \multicolumn{1}{c}{4} & \multicolumn{1}{c}{9} \\
\hline High sensitivity & 0.5 & 0.340 & 0.230 & 0.160 \\
& 1 & 2.33 & 1.58 & 1.26 \\
& 1.5 & 5.49 & 4.10 & 3.15 \\
General purpose & 0.5 & 0.170 & 0.130 & 0.066 \\
& 1 & 1.010 & 0.770 & 0.590 \\
High resolution & 1.5 & 2.73 & 2.06 & 1.59 \\
& 0.5 & 0.1000 & 0.0560 & 0.0500 \\
& 1 & 0.510 & 0.340 & 0.230 \\
& 1.5 & 1.40 & 1.03 & 0.76
\end{tabular}

Septal thickness of collimators was $0.2 \mathrm{~mm}$. 
FIGURE 2. Detection efficiencies (expressed as percentages) for different hole diameters and types of collimator with different septal thicknesses (crystal pixe size, $9 \mathrm{~mm}^{2}$ ). HS = high sensitivity; GP = general purpose; $\mathrm{HR}=$ high resolution.

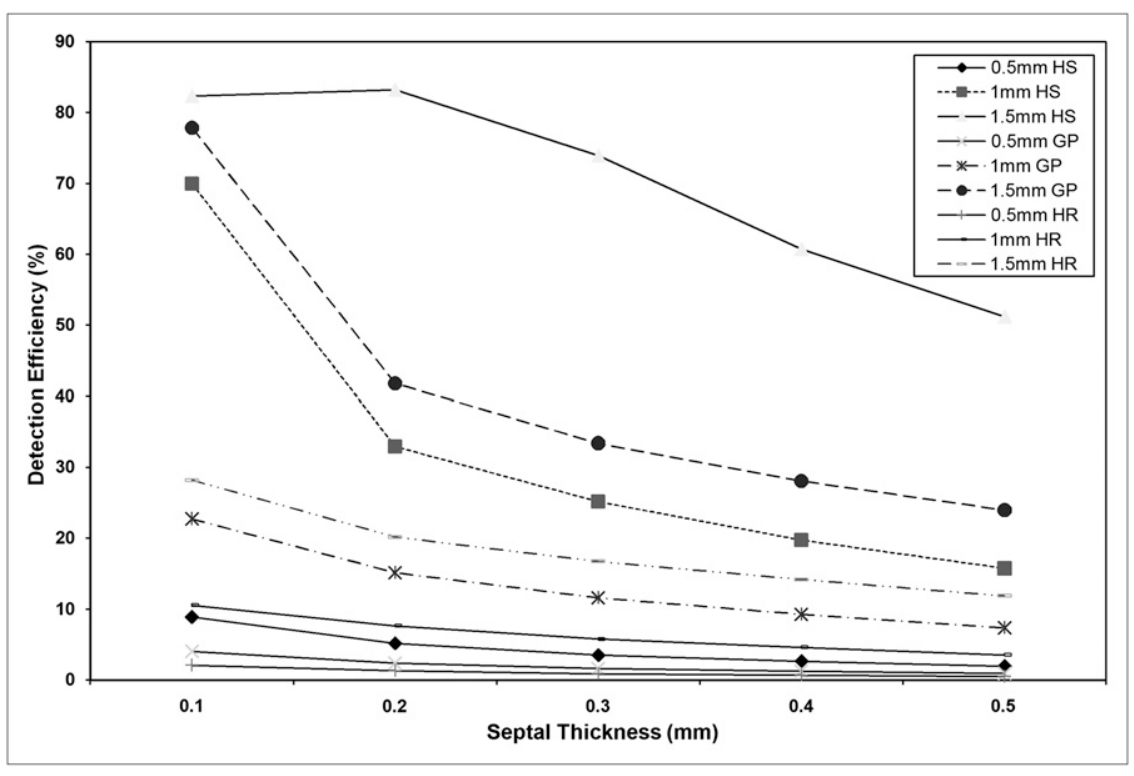

The quality of an image can be described (quantitatively) by its CNR, which directly affects diagnostic and quantitative accuracy. Higher CNR in larger pixel sizes was evident. In most cases, higher CNR was achieved using a collimator hole diameter of $1 \mathrm{~mm}$. Also, increased septal thickness caused CNR to worsen. Table 4 summarizes the results of the comparison of CNR in collimators (septal thickness, $0.2 \mathrm{~mm}$ ) with different crystal pixel sizes, hole diameters, and septal lengths.

Spatial resolution was measured using FWHM (mm). Smaller pixel sizes provided better spatial resolution, and when the collimator hole diameter was increased and septal length was decreased, FWHM deteriorated as expected. Septal thickness variations did not produce much effect on FWHM. Table 5 presents spatial resolution for collimators (septal thickness, $0.2 \mathrm{~mm}$ ) with different crystal pixel sizes, hole diameters, and septal lengths. Our results were in agreement with results of studies performed by Gruber and et al. (13) and Bollini and et al. (8).
In our tests, source-to-collimator distance was $50 \mathrm{~mm}-$ the usual distance defined in scintimammography. The spatial resolution of the system is a function of collimator response and intrinsic resolution. Because collimator resolution decreases when distance from the source increases, extrinsic resolution also decreases (20). Thus, it is expected that smaller distances provide better results.

\section{CONCLUSION}

In this study, we used the MCNP4C general-purpose Monte Carlo code for accurate modeling of ICS effects for different crystal pixel sizes and for defining the best geometry of a hexagonal-hole collimator. We investigated the ICS effect at $140 \mathrm{keV}$ with orthogonal irradiation and the effects of collimator characteristics, with an emphasis on high-resolution breast imaging with scintillators of small pixel size. Optimum crystal pixel size and efficient collimator geometry characteristics, including hole diameter, septal thickness, and length, were achieved and can be used in

TABLE 2

Sensitivity (cpm/MBq) for Collimators with Different Hole Diameters, Septal Thicknesses, and Septal Lengths

\begin{tabular}{|c|c|c|c|c|c|c|}
\hline \multirow[b]{2}{*}{ Type of collimator } & \multirow[b]{2}{*}{ Hole diameter (mm) } & \multicolumn{5}{|c|}{ Septal thickness (mm) } \\
\hline & & 0.1 & 0.2 & 0.3 & 0.4 & 0.5 \\
\hline \multirow[t]{3}{*}{ High sensitivity } & 0.5 & 4,422 & 2,670 & 1,744 & 1,303 & 1,000 \\
\hline & 1 & 35,200 & 16,510 & 12,560 & 9,870 & 8,017 \\
\hline & 1.5 & 41,670 & 41,150 & 37,740 & 30,830 & 25,050 \\
\hline \multirow[t]{3}{*}{ General purpose } & 0.5 & 2,000 & 1,205 & 847 & 659 & 450 \\
\hline & 1 & 11,350 & 7,590 & 5,940 & 4,677 & 3,839 \\
\hline & 1.5 & 38,880 & 20,970 & 16,860 & 14,130 & 12,040 \\
\hline \multirow[t]{3}{*}{ High resolution } & 0.5 & 1,060 & 683 & 468 & 347 & 273 \\
\hline & 1 & 5,250 & 3,804 & 2,935 & 2,330 & 2,031 \\
\hline & 1.5 & 14,000 & 10,230 & 8,359 & 7,064 & 6,027 \\
\hline
\end{tabular}

Crystal pixel size was $4 \mathrm{~mm}^{2}$. 
TABLE 3

Percentage of PDAs in Collimators with Different Crystal Pixel Sizes, Hole Diameters, and Septal Lengths

\begin{tabular}{ccrcc}
\hline & & \multicolumn{3}{c}{ Pixel size $\left(\mathrm{mm}^{2}\right)$} \\
\cline { 3 - 5 } $\begin{array}{c}\text { Type of } \\
\text { collimator }\end{array}$ & $\begin{array}{c}\text { Hole diameter } \\
(\mathrm{mm})\end{array}$ & \multicolumn{1}{c}{1} & 4 & 9 \\
\hline High sensitivity & 0.5 & 26.69 & 66.63 & 80.34 \\
& 1 & 9.13 & 28.62 & 51.95 \\
& 1.5 & 3.41 & 12.62 & 25.27 \\
General purpose & 0.5 & 41.81 & 80.62 & 85.06 \\
& 1 & 16.41 & 46.85 & 75.57 \\
High resolution & 1.5 & 6.76 & 23.86 & 44.48 \\
& 0.5 & 56.84 & 81.05 & 88.57 \\
& 1 & 28.03 & 65.33 & 87.33 \\
& 1.5 & 11.55 & 37.83 & 65.27
\end{tabular}

Septal thickness of collimators was $0.2 \mathrm{~mm}$.

the design of higher-resolution breast tumor imaging systems. Scintillation material characteristics play an important role. Because the effect of ICS and crystal pixel size on camera performance was our assessment goal, we factored attenuation properties of the crystal into the code, and cesium iodide was the crystal of choice with this perspective. We do not claim that cesium iodide is the optimal crystal in all respects, such as decay time, availability, price, and the like. Our experiences confirmed that a crystal pixel size of $2 \times 2$ $\mathrm{mm}$ is optimal, especially for lower ICS fraction and appropriate FWHM. Although smaller pixel size improves spatial resolution, at the same time the misidentification problem could be dominant and therefore image degradation could follow because of an increase of ICS fraction. Collimator geometry selection-including 1-mm hole diameter, $0.2-\mathrm{mm}$ septal thickness, and 21-mm septal length-can provide optimal conditions such as higher CNR and PDA, lower ICS fraction and FWHM, and appropriate DE. Finally, the

TABLE 4

CNRs in Collimators with Different Crystal Pixel Sizes, Hole Diameters, and Septal Lengths

\begin{tabular}{ccccc}
\hline & & \multicolumn{3}{c}{ Pixel size $\left(\mathrm{mm}^{2}\right)$} \\
\cline { 3 - 5 } $\begin{array}{c}\text { Type of } \\
\text { collimator }\end{array}$ & $\begin{array}{c}\text { Hole diameter } \\
(\mathrm{mm})\end{array}$ & 1 & 4 & 9 \\
\hline High sensitivity & 0.5 & 13.49 & 34.43 & 45.16 \\
& 1 & 11.78 & 36.81 & 66.15 \\
& 1.5 & 6.95 & 25.76 & 51.54 \\
General purpose & 0.5 & 14.90 & 29.01 & 29.53 \\
& 1 & 14.27 & 40.82 & 65.76 \\
High resolution & 1.5 & 9.780 & 34.56 & 64.32 \\
& 0.5 & 14.58 & 20.78 & 19.80 \\
& 1 & 17.11 & 40.29 & 54.03 \\
& 1.5 & 11.61 & 38.26 & 65.56
\end{tabular}

Septal thickness of collimators was $0.2 \mathrm{~mm}$.
TABLE 5

Spatial Resolutions (FWHM mm) for Collimators with Different Crystal Pixel Sizes, Hole Diameters, and Septal Lengths

\begin{tabular}{ccccc}
\hline & & \multicolumn{3}{c}{ Pixel size $\left(\mathrm{mm}^{2}\right)$} \\
\cline { 3 - 5 } $\begin{array}{c}\text { Type of } \\
\text { collimator }\end{array}$ & $\begin{array}{c}\text { Hole diameter } \\
(\mathrm{mm})\end{array}$ & 1 & 4 & 9 \\
\hline High sensitivity & 0.5 & 1.626 & 2.192 & 3.036 \\
& 1 & 3.052 & 3.462 & 3.754 \\
General Purpose & 1.5 & 4.704 & 5.179 & 5.395 \\
& 0.5 & 1.232 & 2.042 & 3.010 \\
& 1 & 2.346 & 2.659 & 3.229 \\
High resolution & 1.5 & 3.806 & 3.913 & 4.002 \\
& 0.5 & 1.074 & 2.010 & 3.021 \\
& 1 & 1.686 & 2.317 & 3.055 \\
& 1.5 & 2.572 & 2.880 & 3.395 \\
\hline
\end{tabular}

Septal thickness of collimators was $0.2 \mathrm{~mm}$.

optimal design of a detection system to reach high sensitivity and high resolution in breast tumor imaging is still an open question that demands further work. Exploring other photodetectors such as photodiodes, studying the effects of spacing between crystal pixels (epoxy resin), studying other scintillator and collimator materials, and studying other geometries are possible avenues of further study.

\section{ACKNOWLEDGMENT}

This work has been supported by Tehran University of Medical Sciences.

\section{REFERENCES}

1. Garibaldi F, Cisbani E, Cusanno F, et al. Optimization of compact gamma cameras for breast imaging. Nucl Instr Methods Phys Res A. 2001;471:222-228.

2. Pani R, De Vincentis G, Scopinaro F, et al. Dedicated gamma camera for single photon emission mammography (SPEM). IEEE Nucl Sci Trans. 1998;45:31273133.

3. Pani R, Pellegrini R, De Vincentis G, et al. SPEM: a dedicated camera for scintimammography. Q J Nucl Med. 1996;40:12.

4. Williams MB, Goode AR, Gablis-Reig V, et al. Performance of a PSPMT based detector for scintimammography. Phys Med Biol. 2000;45:781-800.

5. Garibaldi F, Cisbani E, Colilli S, et al. Scintillator and photodetector array optimization for functional breast imaging. Nucl Instr Methods Phys Res A. 2003;497:51-59.

6. Meikle SR, Kench P, Kassiou M, et al. Small animal SPECT and its place in the matrix of molecular imaging technologies. Phys Med Biol. 2005;50:R45-R61.

7. Zaidi H. Recent developments and future trends in nuclear medicine instrumentation. Z Med Phys. 2006;16:5-17.

8. Bollini D, Campanini R, Laconelli N, Riccardi A. A modular description of the geometry in Monte Carlo modeling studies for nuclear medicine. Int $\mathrm{J} \mathrm{Mod}$ Physics C. 2002;13:465-476.

9. Briesmeister JF. MCNP: A General Monte Carlo N-Particle Transport Code Version 4C. Los Alamos, NM: Los Alamos National Laboratory; 2000.

10. Rafecas M, Boning G, Pichler BJ, et al. Inter-crystal scatters in a dual layer, high resolution LSO-APD positron emission tomography. Phys Med Biol. 2003;48: 821-848.

11. Shao Y, Cherry SR, Siegel S, et al. A study of inter-crystal scatter in small scintillator arrays designed for high resolution PET imaging. IEEE Trans Nucl Sci. 1996;43:1938-1944.

12. Stickel JR, Cherry SR. High resolution PET detector designs: modeling components of intrinsic spatial resolution. Phys Med Biol. 2005;50:179-195. 
13. Gruber GJ, Moses WW, Derenzo SE, Wang NW, Beuville E, Ho MH. A discrete scintillation camera module using silicon photodiode readout $\mathrm{CsI}(\mathrm{Tl})$ crystal for breast cancer imaging. IEEE Trans Nucl Sci. 1998;45:1063-1068.

14. Rasouli M, Ay MR, Takavar A, Lashkari S, Loudos G. The influence of intercrystal scattering on detection efficiency of dedicated breast gamma camera: a Monte Carlo study. IFMBE Proceedings. 2009;22:2451-2454.

15. Storm E, Israel HI. Photon Cross-Section from 0.001 to $100 \mathrm{MeV}$ for Elements 1 Through 100. LA-3753. Los Alamos, NM: Los Alamos Scientific Laboratory; 1967.

16. Hubbell JH, Veigele WJ, Briggs EA, Brown RT, Cromer DT, Howerton RJ. Atomic form factors, incoherent scattering functions and photon scattering cross-sections. J Phys Chem Ref Data. 1975;4:471-538.
17. Loudos G, Majewski S, Wojcik R, et al. Performance evaluation of a dedicated camera suitable for dynamic radiopharmaceuticals evaluation in small animals. IEEE Trans Nucl Sci. 2007;54:454-460.

18. Cinti MN, Pani R, Pellegrini R, et al. Tumor SNR analysis in scintimammography by dedicated high contrast imager. IEEE Trans Nucl Sci. 2003;50:1618-1623.

19. Gombia M, Brill AB, Bollini D, Del Guerra A. A simulation and modelling study comparing the performance of a Germanium orthogonal strip detector and an Anger camera. IEEE Trans Nucl Sci. 2002;49:2196-2202.

20. Links JM. Advances in nuclear medicine instrumentation: considerations in the design and selection of an imaging system. Eur J Nucl Med. 1998;25: 1453-1466. 